\begin{tabular}{c|c|c|} 
SELECCIONES MATEMÁTICAS \\
Universidad Nacional de Trujillo \\
ISSN: $2411-1783$ (Online) \\
2021; Vol. 8(2): 333-347.
\end{tabular}

\title{
Application of the principle of the Pontryagin maximum in the solution of a problem of minimum time
}

\section{Aplicación del principio del máximo de Pontryagin en la solución de un problema de tiempo mínimo}

\author{
José Luis Ponte Bejarano® ${ }^{\circledR}$, Juan Carlos Ponte Bejarano® ${ }^{\circledR}$, Alexis Rodríguez Carranza ${ }^{\circledR}$ \\ and Nelson Omar Aragones Salazarī
}

Received, Oct. 01, 2021

Accepted, Dec. 07, 2021

How to cite this article:

Ponte JL, Ponte JC, Rodriguez A, Aragonés N. Application of the principle of the Pontryagin maximum in the solution of a problem of minimum time. Selecciones Matemáticas. 2021;8(2):333-347. http://dx.doi.org/10. 17268 /sel.mat. 2021.02 .10

\begin{abstract}
In the present work, necessary and sufficient optimality conditions are presented for the solution to a minimum time problem. These conditions are established by the principle of the Pontryagin maximum, as a necessary and sufficient condition. In addition, the principle of the Pontryagin maximum is used in the search for the solution to an optimal control problem of mechanical origin.
\end{abstract}

Keywords . Optimal control, ordinary differential equations.

\section{Resumen}

En el presente trabajo se presentan condiciones necesarias y suficientes de optimalidad para la solución a un problema de tiempo mínimo. Estas condiciones son establecidas por el principio del máximo de Pontryagin, como condición necesaria y suficiente. Además, se utiliza el principio del máximo de Pontryagin en la búsqueda de la solución a un problema de control óptimo de origen mecánico.

Palabras clave. Control óptimo, ecuaciones diferenciales ordinarias.

1. Introducción. Los procesos en general, como los técnicos y económicos, que tienen lugar en la práctica son controlables, es decir, pueden ser efectuados de diferentes maneras dependiendo de las decisiones que tome el hombre. En relación con esto, surge la necesidad de buscar el control más adecuado del proceso o, según suele decirse, el control óptimo del proceso. Por ejemplo, uno puede hablar acerca de optimalidad en el sentido de rapidez de acción, es decir, de efectuar el proceso en el menor tiempo posible, con un gasto mínimo de energía u obteniendo un mayor beneficio ([6], [1]). Estos problemas, formulados matemáticamente, corresponden al Cálculo de Variaciones, el cual debe su origen a ellos. Sin embargo, la solución a un gran grupo de estos problemas variacionales, que son importantes en la práctica, están fuera del Cálculo Clásico de Variaciones. Pontryagin y otros de sus colaboradores, teniendo presente las limitaciones del Cálculo Clásico de Variaciones, dieron origen a una teoría, que es una generalización del Cálculo Clásico de Variaciones, a saber, la Teoría de Control óptimo ([3], [4], [5]).

El espíritu de la Teoría de Control óptimo descansa en la manera óptima de gobernar la evolución de un cierto proceso. De esta manera partimos de un proceso, que evoluciona en el tiempo, descrito por medio de una ecuación de estado que describe la dinámica evolutiva de una determinada variable, llamada variable de estado, que varía desde un estado inicial hasta un estado final. La evolución del proceso depende de una

\footnotetext{
*Departamento de Matemáticas, Universidad Nacional de Trujillo, Trujillo, Perú. (jponteb@unitru . edu . pe).

${ }^{\dagger}$ Departamento de Matemáticas, Universidad Nacional de Trujillo, Trujillo, Perú. (jponte@unitru.edu.pe).

${ }_{\ddagger}^{\ddagger}$ Departamento de Matemáticas, Universidad Nacional de Trujillo, Trujillo, Perú. (alrodriguezca@unitru.edu.pe).

$\S$ Departamento de Matemáticas, Universidad Nacional de Trujillo, Trujillo, Perú. (naragones@unitru.edu.pe).
} 
función, llamada control, que puede ser elegida, y con ella es posible influir en la evolución de la variable de estado, de modo tal que haga máximo o mínimo el valor de un funcional objetivo llamado Criterio de Optimalidad ([2], [4], [5]). Ejemplos de funcionales objetivos que se podrían maximizar o minimizar para un cierto proceso son:

- Minimizar el tiempo requerido para transferirse de un estado a otro.

- Maximizar el flujo de beneficios obtenidos a lo largo de un horizonte temporal dado, que comienza en un tiempo $t_{0}$ y termina en $t_{1}$.

Un problema especial en la teoría de Control óptimo consiste en encontrar una función de control, llamada control admisible, con la cual se pueda conducir un cierto proceso, desde un estado inicial hasta un estado final, en el menor tiempo posible. Este problema es llamado problema de tiempo mínimo ([4], [5]]). Establecemos condiciones necesarias y suficientes de optimalidad para este problema. Además, utilizamos el principios del máximo de Pontryagin en la búsqueda de la solución a un problema de control óptimo de origen mecánico.

2. Formulación del problema de control óptimo. Asumamos que un cierto proceso, en el intervalo de tiempo $0 \leq t \leq T$, se describe por el sistema de ecuaciones diferenciales ordinarias:

$$
\frac{d x_{i}}{d t}=f_{i}\left(t, x_{1}, \ldots, x_{n}, u_{1}, \ldots, u_{m}\right), \quad i=1,2, \ldots, n,
$$

o en la forma vectorial

$$
\frac{d x}{d t}=f(t, x, u)
$$

donde $x=\left(x_{1}, \ldots, x_{n}\right)^{T} \in \mathbb{R}^{n}, f=\left(f_{1}, \ldots, f_{n}\right)^{T} \in \mathbb{R}^{n}$ con $f_{1}, \ldots, f_{n}$ continuas junto con sus derivadas parciales respecto de $x_{1}, \ldots, x_{n}, u=\left(u_{1}, \ldots, u_{m}\right)^{T} \in U$, y $U$ es un conjunto cerrado y acotado en el espacio $\mathbb{R}^{m}$. El vector $x$ se denomina vector de estado, y las variables $x_{1}, \ldots, x_{n}$ se denominan variables de fase o de estado. La función vectorial $u(t)$, definida sobre $[0, T]$, con rango en $U$ se denomina control. Las funciones $u_{1}(t), u_{2}(t), \ldots, u_{m}(t)$ se denominan variables de control. El conjunto $U$ se denomina región de control. Cada control $u(t)$ continua a trozos se denomina control admisible.

Consideremos el estado inicial:

$$
x(0)=x^{0} .
$$

Entonces, para cada control admisible $u(t)$, el problema de Cauchy formado por (2.1) y (2.2) tiene solución única $x(t)$ denominada trayectoria o curva de fase.

Sea que para un conjunto de controles admisibles $u(t)$ y sus correspondientes trayectorias $x(t)$, está definido el funcional objetivo, llamado criterio de optimalidad, por:

$$
I(u)=\int_{0}^{T} G(t, x(t), u(t)) d t+F(x(T)),
$$

donde $G: D^{\prime} \subset \mathbb{R} \times \mathbb{R}^{n} \times \mathbb{R}^{m} \rightarrow \mathbb{R}$ y $F: D^{\prime \prime} \subset \mathbb{R}^{n} \rightarrow \mathbb{R}$ poseen derivadas parciales de primer orden continuas. Se plantea el problema: hallar un control admisible $u^{*}(t)$, tal que para él y para su trayectoria correspondiente $x^{*}(t)$ el funcional objetivo (2.3) alcance su valor extremo:

$$
I\left(u^{*}\right)=\operatorname{máx}_{u \in U} I(u) \quad\left(\text { ó } I\left(u^{*}\right)=\operatorname{mín}_{u \in U} I(u)\right) .
$$

Definición 2.1. El control admisible $u^{*}(t)$, que soluciona el problema expuesto anteriormente, se denomina control óptimo, $x^{*}(t)$ trayectoria óptima y el par de funciones $\left(u^{*}(t), x^{*}(t)\right)$ proceso óptimo.

El problema de control óptimo expuesto lineas anteriores se puede expresar como:

$$
\begin{aligned}
& \operatorname{máx}_{u} I(u)=\int_{0}^{T} G(t, x(t), u(t)) d t+F(x(T)) \\
& \text { sujeto a: } \frac{d x}{d t}=f(t, x, u), \\
& \text { con: } x(0)=x^{0} \\
& u(t) \in U .
\end{aligned}
$$

Cuando el funcional objetivo es del tipo

$$
I(u)=F(x(T)),
$$

se dice que está en la forma de Mayer. Así, el ploblema de control óptimo con funcional objetivo de este tipo recibe el nombre de problema de Mayer. Además, en lugar del problema de maximización se puede considerar el problema de minimización. 
2.1. Función de Hamilton. Consideremos el sistema de ecuaciones (2.1) para el caso autónomo:

$$
\frac{d x_{i}}{d t}=f_{i}(x, u), i=1,2, \ldots, n,
$$

y el sistema de ecuaciones en las funciones auxiliares $\psi_{1}, \ldots, \psi_{n}$ :

$$
\frac{d \psi_{i}}{d t}=-\sum_{\alpha=1}^{n} \frac{\partial f_{\alpha}(x, u)}{\partial x_{i}} \psi_{\alpha}, i=1,2, \ldots, n .
$$

Si elegimos un control admisible $u(t), 0 \leq t \leq T$, y tenemos la respectiva trayectoria de fase $x(t)$ del sistema (2.4) con condición inicial $x(0)=x^{0}$, el sistema (2.5) toma la forma:

$$
\frac{d \psi_{i}}{d t}=-\sum_{\alpha=1}^{n} \frac{\partial f_{\alpha}(x(t), u(t))}{\partial x_{i}} \psi_{\alpha}, i=1,2, \ldots, n .
$$

Por lo tanto, para cada condición inicial, este sistema admite una única solución

$$
\psi=\left(\psi_{1}, \ldots, \psi_{n}\right),
$$

para los $\psi_{i}$ definidos sobre el intervalo $0 \leq t \leq T$, en el cual $u(t)$ y $x(t)$ están definidas.

Observación 2.1. La solución del sistema (2.6) es de la forma $\psi=\left(\psi_{1}, \ldots, \psi_{n}\right)$, donde los $\psi_{i}$ son funciones continuas que tienen derivadas parciales continuas, respecto de $t$, en todas partes, excepto en un número finito de puntos (los puntos de discontinuidad de $u(t)$ ).

Ahora combinemos los sistemas (2.4) y (2.5). Para ello consideraremos una nueva función, denominada función de Hamilton, la que se define a continuación.

Definición 2.2. La función $H$, de las variables $x_{1}, \ldots, x_{n}, \psi_{1}, \ldots, \psi_{n}, u_{1}, \ldots, u_{m}$, definida por:

$$
H(x, u, \psi)=\langle\psi, f(x, u)\rangle=\sum_{\alpha=1}^{n} \psi_{\alpha} f_{\alpha}(x, u),
$$

se denomina función de Hamilton o Hamiltoniano.

Mediante esta función, los sistemas (2.4) y (2.5) pueden ser reescritos en forma del siguiente sistema Hamiltoniano:

$$
\begin{gathered}
\frac{d x_{i}}{d t}=\frac{\partial H}{\partial \psi_{i}}, i=1,2, \ldots, n, \\
\frac{d \psi_{i}}{d t}=-\frac{\partial H}{\partial x_{i}}, i=1,2, \ldots, n .
\end{gathered}
$$

Así, tomando un control admisible $u(t), 0 \leq t \leq T$, y la condición inicial $x(0)=x^{0}$, podemos encontrar (satisfaciendo (2.7)) la correspondiente trayectoria $x(t)=\left(x_{1}(t), \ldots, x_{n}(t)\right)$.

3. Formulación del problema de tiempo mínimo. Asumamos que un cierto proceso se describe por el sistema lineal de ecuaciones diferenciales ordinarias:

$$
\frac{d x_{i}}{d t}=\sum_{k=1}^{n} a_{i k}(t) x_{k}+\sum_{l=1}^{m} b_{i l}(t) u_{l}, \quad i=1, \ldots, n,
$$

donde $x=\left(x_{1}, \ldots, x_{n}\right)^{T}$ es el vector de estado; $u=\left(u_{1}, \ldots, u_{m}\right)^{T}$ es el control y para cualesquier momentos considerados de tiempo $t$ el valor del control $u(t)$ pertenece a la región de control $U$, la cual asumimos convexa cerrada y acotada en el espacio $\mathbb{R}^{m}$.

El problema de control óptimo, llamado de tiempo mínimo, consiste en lo siguiente: entre todos los controles admisibles, determinar el óptimo, es decir, aquel para el cual la transición del proceso, desde un estado inicial $x^{0}$ hasta un estado final $x^{1}$, se realiza en un tiempo mínimo.

Consideremos $A=\left(a_{i k}(t)\right)_{n \times n}, B=\left(b_{i l}(t)\right)_{n \times m}$, momento de tiempo inicial $t=0$, momento de tiempo final $t_{f}$, y sea que el estado final es el origen de coordenadas: $x^{1}=0$. Entonces, el proceso puede escribirse en forma del siguiente problema de contorno:

$$
\left\{\begin{array}{l}
\frac{d x}{d t}=A x+B u \\
x(0)=x^{0}, \quad x\left(t_{f}\right)=0 .
\end{array}\right.
$$


A cada control admisible $u(t)$, con valores en $U$, le corresponde una solución $x(t)$ del problema de contorno (3.1), una curva en el espacio de fase determinada por esta solución (trayectoria), y un tiempo de transición del proceso $t_{f}(u)$. El problema de tiempo mínimo consiste en determinar entre todos los controles admisibles el control óptimo $u^{*}(t)$, para el cual el tiempo de transición del proceso $t_{f}\left(u^{*}\right)$, por la trayectoria correspondiente, es mínimo:

$$
t_{f}\left(u^{*}\right)=\min _{u \in U} t_{f}(u)
$$

En lo sigue asumiremos que $U$ contiene al origen de coordenadas $u=0$ del espacio $\mathbb{R}^{m}$. El problema de tiempo mínimo se puede formular de la siguiente manera:

$$
\begin{gathered}
\min _{u \in U} t_{f}(u) \\
\text { sujeto a: } \frac{d x}{d t}=A x+B u, \\
\text { con: } x(0)=x^{0}, x\left(t_{f}\right)=0, \\
u(t) \in U,
\end{gathered}
$$

donde $t_{f}(u)$ es el tiempo de transición del proceso, $A=\left(a_{i k}(t)\right)_{n \times n}, B=\left(b_{i l}(t)\right)_{n \times m}, x^{0}$ y 0 son los estados de fase inicial y final, respectivamente.

4. Principio del máximo de Pontryagin. Antes de mencionar los principios del máximo de Pontryagin se definirá la esfera de accesibilidad y el poliedro de posición general.

Definición 4.1. Esfera de Accesibilidad en el tiempo $T$ se denomina al conjunto $S_{T}$ de todos los estados de fase $x \in \mathbb{R}^{n}$, para cada uno de los cuales existe un control admisible que traslada este estado inicial hasta el estado final $x=0$ por la trayectoria correspondiente en un tiempo que no supera a $T$.

Observación 4.1. Dos propiedades de la esfera de accesibilidad son las siguientes:

a) Propiedad 1: La esfera de accesibilidad $S_{T}$ es un conjunto convexo en $\mathbb{R}^{n}$.

b) Propiedad 2: Todo punto de la Esfera de Accesibilidad $S_{T}$, para el cual no existe un control admisible que traslade un estado desde este punto hasta el origen de coordenadas en un tiempo menor que $T$, pertenece a la frontera de la Esfera de accecibilidad $S_{T}$.

Cuando las matrices $A$ y $B$ que aparecen en el sistema de ecuaciones

$$
\frac{d x}{d t}=A x+B u
$$

no dependen de $t$, estas pueden ser entendidas como operadores lineales, es decir, la matriz $A$ puede ser entendida como el operador $A: \mathbb{R}^{n} \rightarrow \mathbb{R}^{n}$ y la matriz $B$ como el operador $B: \mathbb{R}^{m} \rightarrow \mathbb{R}^{n}$.

Definición 4.2. Un poliedro $U \subset \mathbb{R}^{m}$ se denomina poliedro de posición general si para cualquier arista $w$ de $U$ el vector Bw tiene la propiedad que no pertenece a ningún subespacio propio de $\mathbb{R}^{n}$, invariante respecto del operador $A$.

Observación 4.2. Para el caso lineal, la función de Hamilton $H(x, u, \psi)$ tiene la forma:

$$
H(x, u, \psi)=\langle\psi, A x+B u\rangle=\langle\psi, A x\rangle+\langle\psi, B u\rangle,
$$

donde el segundo sumando $\langle\psi, B u\rangle$, considerada como función de u, se denomina función de Pontryagin y es dada por:

$$
F(u)=\langle\psi, B u\rangle
$$

Además, la función $\psi(t)$ toma la forma:

$$
\frac{d \psi}{d t}=-A^{T} \psi
$$

Consideremos el problema de Mayer para el caso cuando los términos derechos del sistema (2.1) no depende de $t$ explicitamente. Y asumamos que el criterio de optimalidad (2.3) toma la forma

$$
I(u)=c_{1} x_{1}(T)+\cdots+c_{n} x_{n}(T)=\langle c, x(T)\rangle
$$

y para el control óptimo $u^{*}$,

$$
I\left(u^{*}\right)=\operatorname{máx}_{u \in U} I(u) \text {. }
$$


Teorema 4.1. [4] Si $\left(u^{*}(t), x^{*}(t)\right)$ es un proceso óptimo del problema de Mayer considerado, $\psi(t)$ es solución del problema de Cauchy

$$
\frac{d \psi}{d t}=-\mathcal{J}^{T} \psi, \quad \psi(T)=c
$$

donde

$$
\mathcal{J}=\left[\begin{array}{llr}
\frac{\partial f_{1}}{\partial x_{1}} & \cdots & \frac{\partial f_{1}}{\partial x_{n}} \\
\cdot & \cdots & \cdot \\
\cdot & \cdots & \cdot \\
\cdot & \cdots & \cdot \\
\frac{\partial f_{n}}{\partial x_{1}} & \cdots & \frac{\partial f_{n}}{\partial x_{n}}
\end{array}\right]
$$

$y H(x, u, \psi)=\langle\psi, f(x, u)\rangle$ la función de Hamilton. Entonces tiene lugar la igualdad

$$
H\left(x^{*}, u^{*}, \psi\right)=\operatorname{máx}_{u \in U} H\left(x^{*}, u, \psi\right) .
$$

$\left[\right.$ Cuando $I\left(u^{*}\right)=\operatorname{mín}_{u \in U} I(u)$, tiene lugar la igualdad:

$$
\left.H\left(x^{*}, u^{*}, \psi\right)=\min _{u \in U} H\left(x^{*}, u, \psi\right)\right]
$$

Teorema 4.2. [4] Asumamos que un cierto proceso se describe mediante el problema de contorno (3.1). Sea $\psi(t)$ solución del problema de Cauchy:

$$
\frac{d \psi}{d t}=-A^{T} \psi, \quad \psi(0)=c
$$

para algún c (la naturaleza de c será aclarada durante la demostración), y $H(x, u, \psi)=\langle\psi, A x+B u\rangle$ la función de Hamilton. Si $\left(u^{*}(t), x^{*}(t)\right)$ es un proceso óptimo (para el cual el tiempo $T=t_{f}\left(u^{*}\right)$ de transición del proceso correspondiente, según la trayectoria óptima $x^{*}(t)$, satisface la condición (3.2)), se tendrá:

$$
H\left(x^{*}, u^{*}, \psi\right)=\operatorname{máx}_{u \in U} H\left(x^{*}, u, \psi\right)
$$

Demostración: Para la demostración transformaremos el problema de tiempo mínimo al problema de Mayer. Por la propiedad 2 de $S_{T}, x^{0}$ se encuentra en la frontera de la Esfera de Accesibilidad $S_{T}$, ya que si fuese punto interior se podría llegar al origen de coordenadas en un tiempo menor que $T$ y esto contradice el hecho de que $u^{*}(t)$ es un control óptimo. Por otra parte, es claro que $x^{0}$ no puede estar fuera de $S_{T}$. Además, por la propiedad 1 de $S_{T}$, la Esfera de Accesibilidad $S_{T}$ es convexa. Por lo tanto, en el punto $x^{0}$ puede determinarse un hiperplano soporte a $S_{T}$ (de modo que $S_{T}$ se encuentra a un lado de este hiperplano). En nuestro caso ocurre lo de Figura 4.1.

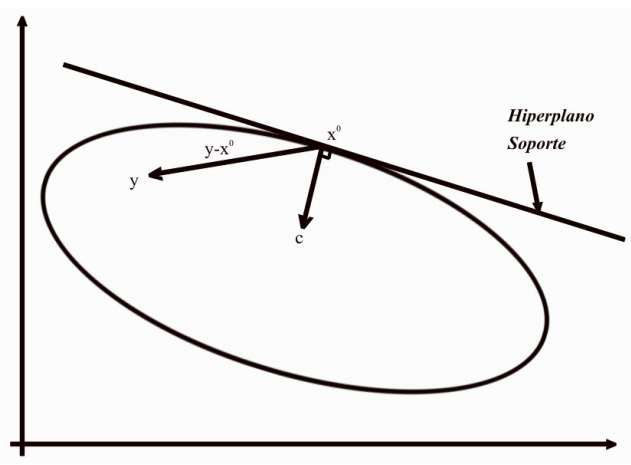

Figura 4.1: La Esfera $\mathbf{S}_{T}$ a un lado del Hiperplano Soporte. 
Para cada $y \in S_{T}$ existe un control admisible $u(t)$ que traslada el estado $y$ hasta el origen de coordenadas en un tiempo $t_{f}(u) \leq T$. Si consideramos $u(t) \equiv 0$ en $\left[t_{f}(u), T\right]$ (el cual siempre es posible pues $U$ contiene al origen de coordenadas de $\mathbb{R}^{m}$ ) entonces se puede construir un control admisible $u(t)$ que traslada el estado $y$ hasta el origen de coordenadas en el tiempo $T$. Correspondiente a este control admisible $u(t)$ se obtiene la trayectoria $x(t)$ con la siguiente propiedad:

$$
x(0)=y \in S_{T} \text { y } x(T)=0 .
$$

Ahora consideremos el desplazamiento por trayectorias, no desde los puntos de $S_{T}$ hacia el origen de coordenadas, sino en sentido contrario: desde el origen de coordenadas hacia los puntos de $S_{T}$. De este modo, por la trayectoria $x^{*}(t)$ se llega desde $x^{0}$ hasta el origen de coordenadas en el tiempo T, y desde otro punto de $S_{T}$, como un punto frontera de $S_{T}$ o un punto interior de $S_{T}$, se llega hasta el origen de coordenadas, por la trayectoria correspondiente al punto elegido en $S_{T}$ en el tiempo $T$. En relación con esto hagamos el siguiente cambio de variables:

$$
t=T-t^{\prime}, \quad x(t)=x\left(T-t^{\prime}\right)=: x^{\prime}\left(t^{\prime}\right), \quad u(t)=u\left(T-t^{\prime}\right)=: u^{\prime}\left(t^{\prime}\right) .
$$

Entonces:

$$
\frac{d x^{\prime}}{d t^{\prime}}=\frac{d x}{d t} \cdot \frac{d t}{d t^{\prime}}=-\frac{d x}{d t}
$$

y el sistema del problema de contorno (3.1) toma la siguente forma:

$$
\frac{d x^{\prime}}{d t^{\prime}}=-A x^{\prime}-B u^{\prime}
$$

La condición de contorno $x(T)=0$, para cualquier trayectoria $x(t)$ correspondiente a los puntos de $S_{T}$, se transforma en la condición $x^{\prime}(0)=0$, y la condición inicial del problema de minimización del tiempo $x(0)=x^{0}$, para el proceso óptimo $\left(u^{*}(t), x^{*}(t)\right)$, toma la forma $x^{\prime *}(T)=x^{0}$. Y para las trayectorias $x(t)$, correspondiente a los puntos $y \in S_{T}$ diferentes de $x^{0}$, se tiene $y=x^{\prime}(T) \neq x^{0}, x^{\prime}(T) \in S_{T}$. Si denotamos por $c$ la normal al hiperplano soporte a $S_{T}$ en el punto $x^{0}$, dirigido hacia el interior de la Esfera de Accesibilidad $S_{T}$, se cumple:

$$
\left\langle x^{\prime}(T)-x^{\prime *}(T), c\right\rangle \geq 0 .
$$

Esta desigualdad se verifica para cada $x^{\prime}(T) \in S_{T}$, y por ende para cada control admisible $u^{\prime}\left(t^{\prime}\right)$ que traslada el estado $x^{\prime}(0)=0$ hasta el estado $x^{\prime}(T)$. Como cada control admisible $u^{\prime}\left(t^{\prime}\right)$ traslada $x^{\prime}(0)=0$ hasta $x^{\prime}(T)$ por la trayectoria $x^{\prime}\left(t^{\prime}\right)$, tenemos:

$$
\left\langle x^{\prime}(T)-x^{\prime *}(T), c\right\rangle \geq 0, \quad \forall u^{\prime}, u^{\prime}\left(t^{\prime}\right) \in U
$$

o que es lo mismo:

$$
\left\langle x^{* *}(T), c\right\rangle=\operatorname{mín}_{u^{\prime} \in U}\left\langle x^{\prime}(T), c\right\rangle .
$$

De esta manera se llega al problema de Mayer: para el sistema (4.5), en el segmento de tiempo [0,T], con condición inicial $x^{\prime}(0)=0$, se requiere determinar el proceso óptimo $\left(u^{\prime *}\left(t^{\prime}\right), x^{\prime *}\left(t^{\prime}\right)\right)$ que minimiza la funcional $\left\langle x^{\prime}(T), c\right\rangle$.

Se observa lo siguiente:

1. $\left(u^{\prime *}\left(t^{\prime}\right), x^{\prime *}\left(t^{\prime}\right)\right)$ es un proceso óptimo del problema de Mayer, pues $\left(u^{\prime *}\left(t^{\prime}\right), x^{\prime *}\left(t^{\prime}\right)\right)$ es tal que:

$$
\left\langle x^{*}(T), c\right\rangle=\min _{u^{\prime} \in U}\left\langle x^{\prime}(T), c\right\rangle .
$$

2. $\psi^{\prime}\left(t^{\prime}\right)$ es solución de problema de Cauchy:

$$
\frac{d \psi^{\prime}}{d t^{\prime}}=-\mathcal{J}^{T} \psi^{\prime}, \psi^{\prime}(T)=c,
$$

donde

$$
\mathcal{J}=\left[\begin{array}{llr}
-a_{11} & \ldots & -a_{1 n} \\
\cdot & \ldots & \cdot \\
\cdot & \ldots & \cdot \\
\cdot & \ldots & \cdot \\
-a_{n 1} & \ldots & -a_{n n}
\end{array}\right]
$$


es la matriz Jacobiana de la función $f\left(x^{\prime}, u^{\prime}\right)=-A x^{\prime}-B u^{\prime}$.

En efecto:

Debido al cambio de variable $t=T-t^{\prime}$, se tiene:

$$
\psi(t)=\psi\left(T-t^{\prime}\right)=: \psi^{\prime}(t)
$$

Usando el cambio de variable anterior y el hecho de que $\psi(t)$ es solución del problema de Cauchy:

$$
\frac{d \psi}{d t}=-A^{T} \psi, \psi(0)=c,
$$

se llega a lo siguiente:

$$
\frac{d \psi^{\prime}}{d t^{\prime}}=-\mathcal{J}^{T} \psi^{\prime}, \psi^{\prime}(T)=c,
$$

donde $\mathcal{J}=-A$ es la matriz Jacobiana de la función $f\left(x^{\prime}, u^{\prime}\right)=-A x^{\prime}-B u^{\prime}$. Como $\psi(t)$ es solución del problema de Cauchy:

$$
\frac{d \psi}{d t}=-A^{T} \psi, \psi(0)=c,
$$

entonces, debido al cambio de variable anterior, $\psi^{\prime}\left(t^{\prime}\right)$ es solución del problema de Cauchy:

$$
\frac{d \psi^{\prime}}{d t^{\prime}}=-\mathcal{J}^{T} \psi^{\prime}, \psi^{\prime}(T)=c
$$

Luego, usando el Teorema 4.1 tenemos:

$$
H^{\prime}\left(x^{\prime *}, u^{\prime *}, \psi^{\prime}\right)=\min _{u^{\prime} \in U} H^{\prime}\left(x^{\prime *}, u^{\prime}, \psi^{\prime}\right),
$$

donde $H^{\prime}\left(x^{\prime}, u^{\prime}, \psi^{\prime}\right)=\left\langle\psi^{\prime},-A x^{\prime}-B u^{\prime}\right\rangle$ es la función de Hamilton.

Retornando a las variables originales $t, x, u$, el problema de Cauchy (4.6), para $\psi^{\prime}\left(t^{\prime}\right)$, se transforma en el problema de Cauchy (4.3) (ver la formulación de Teorema) para $\psi(t)=\psi\left(T-t^{\prime}\right)$. Como $H(x, u, \psi)=$ $\langle\psi, A x+B u\rangle$, entonces:

$$
H^{\prime}\left(x^{\prime}, u^{\prime}, \psi^{\prime}\right)=-H(x, u, \psi)
$$

pues $\psi^{\prime}\left(t^{\prime}\right)=\psi(t), x^{\prime}\left(t^{\prime}\right)=x(t)$ y $u^{\prime}\left(t^{\prime}\right)=u(t)$. Usando esta igualdad, se tiene:

$$
\begin{aligned}
H^{\prime}\left(x^{\prime *}, u^{\prime *}, \psi^{\prime}\right) & =\min _{u^{\prime} \in U} H^{\prime}\left(x^{\prime *}, u^{\prime}, \psi^{\prime}\right) \\
\Longleftrightarrow H\left(x^{*}, u^{*}, \psi\right) & =-\min _{u \in U}-H\left(x^{*}, u, \psi\right) \\
\Longleftrightarrow H\left(x^{*}, u^{*}, \psi\right) & =\operatorname{máx}_{u \in U} H\left(x^{*}, u, \psi\right) .
\end{aligned}
$$

Así, el punto mínimo $u^{\prime *}$ para $H^{\prime}\left(x^{\prime *}, u^{\prime}, \psi^{\prime}\right)$ será el punto máximo $u^{*}$ para $H\left(x^{*}, u, \psi\right)$. De este modo, se tiene:

$$
H\left(x^{*}, u^{*}, \psi\right)=\operatorname{máx}_{u \in U} H\left(x^{*}, u, \psi\right)
$$

que es la igualdad (4.4).

El teorema 4.2 se conoce como el principio del máximo de Pontryagin como condición necesaria de optimalidad del control para el problema de tiempo mínimo. Ahora, se enunciará el principio del máximo de Pontrygin como condición suficiente de optimalidad del control para el problema de tiempo mínimo. Antes de ello se enunciarán algunos lemas.

Lema 4.1. Si u(t) es un control admisible arbitrario, $x(t)$ la solución del sistema correspondiente $\frac{d x}{d t}=A x+B u y \psi(t)$ la solución del sistema $\frac{d \psi}{d t}=-A^{T} \psi$ entonces:

$$
\frac{d}{d t}\langle\psi, x\rangle=\langle\psi, B u\rangle
$$


Lema 4.2. Si $v$ es algún vector constante dado en $\mathbb{R}^{n}, \psi(t)$ una solución no trivial en $\left[t_{1}, t_{2}\right]$ del sistema $\frac{d \psi}{d t}=-A^{T} \psi y \forall t \in\left[t_{1}, t_{2}\right]$ se tiene $\langle\psi(t), v\rangle=0$, entonces $v$ pertenece a un subespacio propio invariante de $\mathbb{R}^{n}$ respecto del operador $A$.

Lema 4.3. La función de Pontryagin $F(u)$, como función de $u$, no identica a una constante alcanza su máximo respecto de $u$ en el punto $u^{*}$ de la frontera del poliedro $U \subset \mathbb{R}^{m}$. Si este punto de máximo $u^{*}$ no se encuentra en un vértice del poliedro $U$, sino que es punto interior de alguna arista o faceta (cara), entonces la función de Pontryagin será constante en la arista o faceta correspondiente.

Teorema 4.3. [5] Consideremos que:

1. Un cierto proceso se describe mediante el sistema $\frac{d x}{d t}=A x+B u$ con coeficientes constantes $y$ condiciones de contorno $x(0)=x^{0}, x\left(t_{f}\right)=0$ y sobre el vector de control se asume la siguiente la restricción: $u \in U$.

2. U es un poliedro convexo de posición general que contiene al origen de coordenadas $u=0$, pero no como un vértice.

3. $\psi(t)$ es una solución no trivial del sistema

$$
\frac{d \psi}{d t}=-A^{T} \psi
$$

y $H(x, u, \psi)=\langle\psi, A x+B u\rangle$ la función de Hamilton.

4. Para algún proceso admisible $\left(u^{*}(t), x^{*}(t)\right)$ tiene lugar el Principio del Máximo de Pontryagin:

$$
H\left(x^{*}, u^{*}, \psi\right)=\operatorname{máx}_{u \in U} H\left(x^{*}, u, \psi\right) .
$$

Entonces $\left(u^{*}(t), x^{*}(t)\right)$ es el proceso óptimo del problema de tiempo mínimo planteado:

$$
T=t_{f}\left(u^{*}\right)=\min _{u \in U} t_{f}(u) .
$$

Demostración: Plantearemos la demostración por el absurdo. Supongamos que el proceso $\left(u^{*}(t), x^{*}(t)\right)$, que satisface el Principio del máximo de Pontryagin, no es un proceso óptimo, entonces existe un proceso $(u(t), x(t))$ tal que $x(0)=x^{0}, x(\theta)=0$, donde $0<\theta<T$. Apartir de esto lleguemos a una contradición con una condición del teorema, a saber, que $U$ es un poliedro de posición general.

Por el lema 4.1 se tiene:

$$
\left\{\begin{array}{l}
\frac{d}{d t}\left\langle\psi, x^{*}\right\rangle=\left\langle\psi, B u^{*}\right\rangle \text { en }[0, T] ; \\
\frac{d}{d t}\langle\psi, x\rangle=\langle\psi, B u\rangle \text { en }[0, \theta] .
\end{array}\right.
$$

Integrando cada una de estas igualdades en el segmento $[0, \theta]$ :

$$
\begin{gathered}
\left\langle\psi(\theta), x^{*}(\theta)\right\rangle-\left\langle\psi(0), x^{*}(0)\right\rangle=\int_{0}^{\theta}\left\langle\psi(t), B u^{*}(t)\right\rangle d t, \\
\langle\psi(\theta), x(\theta)\rangle-\langle\psi(0), x(0)\rangle=\int_{0}^{\theta}\langle\psi(t), B u(t)\rangle d t .
\end{gathered}
$$

Restando de la primera igualdad la segunda igualdad, considerando que $x^{*}(0)=x(0)=x^{0}$ y $x(\theta)=$ 0 , se obtiene:

$$
\left\langle\psi(\theta), x^{*}(\theta)\right\rangle=\int_{0}^{\theta}\left[\left\langle\psi, B u^{*}\right\rangle-\langle\psi, B u\rangle\right] d t .
$$
tiene:

Como $\left(u^{*}(t), x^{*}(t)\right)$ es un proceso admisible que satisface el Principio del Máximo de Pontryagin, se

$$
H\left(x^{*}, u^{*}, \psi\right) \geq H\left(x^{*}, u, \psi\right) \forall u \in U,
$$

es decir:

$$
\left\langle\psi, B u^{*}\right\rangle \geq\langle\psi, B u\rangle \forall u \in U .
$$


De esto y de (4.8), se obtiene:

$$
\left\langle\psi(\theta), x^{*}(\theta)\right\rangle \geq 0
$$

Integrando (4.7) en el segmento $[\theta, T]$, se obtiene:

$$
\left\langle\psi(T), x^{*}(T)\right\rangle-\left\langle\psi(\theta), x^{*}(\theta)\right\rangle=\int_{\theta}^{T}\left\langle\psi(t), B u^{*}(t)\right\rangle d t .
$$

Como $\left\langle\psi, B u^{*}\right\rangle \geq\left.\langle\psi, B u\rangle\right|_{u=0}=0$ en $[0, T]$ y $[\theta, T] \subset[0, T]$, se tiene:

$$
\left\langle\psi, B u^{*}\right\rangle \geq 0 \text { en }[\theta, T] \text {. }
$$

Luego:

$$
\int_{\theta}^{T}\left\langle\psi, B u^{*}\right\rangle d t \geq 0
$$

De esto, de (4.10) y considerando que $x^{*}(T)=0$, se tiene:

$$
\left\langle\psi(\theta), x^{*}(\theta)\right\rangle \leq 0
$$

De (4.9) y (4.11) se sigue que: $\left\langle\psi(\theta), x^{*}(\theta)\right\rangle=0$. Entonces de (4.10), considerando $x^{*}(T)=0$, se tiene:

$$
\int_{\theta}^{T}\left\langle\psi, B u^{*}\right\rangle d t=0
$$

De esto y recordando que $\left\langle\psi(t), B u^{*}(t)\right\rangle \geq 0$ en $[\theta, T]$, se obtiene:

$$
\left\langle\psi(t), B u^{*}(t)\right\rangle=0, \quad \forall t \in[\theta, T]
$$

es decir, la función de Pontryagin $F(u)$ alcanza su valor máximo, respecto de $u$, igual a cero: $F\left(u^{*}\right)=0$. Puesto que $F(u)=0$ para $u=0$, y $u=0$, por condición del teorema, no se encuentra en un vértice del poliedro $U$, entonces $\forall t \in[\theta, T]$ el punto máximo $u^{*}=0$, para la función de Pontryagin, es un punto interior de una arista o faceta de poliedro $U$. Luego, por el lema 4.3 , la función de Pontryagin es constante en esta arista o faceta. Por lo tanto, existen dos puntos $u^{\prime}$ y $u^{\prime \prime}$ que pertenecen a una misma arista en los cuales la función de Pontryagin toma un mismo valor para todos los $t \in[\theta, T]$, es decir: $\left\langle\psi(t), B u^{\prime}\right\rangle \equiv\left\langle\psi(t), B u^{\prime \prime}\right\rangle$ ó $\left\langle\psi(t), B\left(u^{\prime}-u^{\prime \prime}\right)\right\rangle \equiv 0$ en el segmento $[\theta, T]$. Luego, por el lema 4.2, el vector $B\left(u^{\prime}-u^{\prime \prime}\right)$ pertenece a un subespacio propio de $\mathbb{R}^{n}$ invariante respecto del operador $A$. Este hecho contradice la condición que $U$ es un poliedro de posición general. De este modo se concluye la demostración del teorema.

El siguiente teorema establece que el control óptimo es contante por partes y que toma su valor en los vértices de $U$.

Teorema 4.4. [5] Consideremos que:

1. $u^{*}(t)$ es un control óptimo del problema de tiempo mínimo para el sistema lineal con coeficientes constantes $\frac{d x}{d t}=A x+B u$ y condiciones de contorno $x(0)=x^{0}, x\left(t_{f}\right)=0$ y sobre el vector de control se asume la siguiente restricción: $u \in U$.

2. U es un poliedro convexo de posición general con vértices $u^{1}, u^{2}, \ldots, u^{N}$, que contiene al origen de coordenadas $u=0$, pero no como un vértice.

3. $\psi(t)$ es una solución no trivial del sistema $\frac{d \psi}{d t}=-A^{T} \psi$.

4. T es el tiempo que toma la transición del proceso sobre la trayectoria óptima.

Entonces existe un número finito de momentos de tiempo $t_{0}, t_{1}, \ldots, t_{r}$ tales que $0=t_{0}<t_{1}<\ldots<$ $t_{r}=T ; \forall i, i=1, \ldots, r, u^{*}(t)$ es constante en $\left(t_{i-1}, t_{i}\right)$ y toma su valor en un vértice de $U$, a saber, $u^{1}, u^{2}, \ldots, u^{N}$.

Demostración: Del teorema 4.3 sabemos que la función de Pontryagin $F(u)=\langle\psi, B u\rangle$ alcanza su máximo en el control óptimo $u^{*}: F\left(u^{*}\right)=\operatorname{máx}_{u \in U} F(u)$, y que si $F(u) \not \equiv$ constante entonces los valores de $u^{*}(t)$ pertenecen a la frontera del poliedro $U$ para todo $t \in[0, T]$, según el lema 4.3.

Mostremos inicialmente que sólo para un número finito de momentos de tiempo $t_{0}, t_{1}, \ldots, t_{r}$ los valores $u^{*}\left(t_{0}\right), u^{*}\left(t_{1}\right), \ldots, u^{*}\left(t_{r}\right)$ se encuentran en las aristas o facetas del poliedro y no en sus vértices.

Asumamos que estos momentos de tiempo constituyen un conjunto infinito. Puesto que en el poliedro $U$ hay un número finito de facetas, entonces para una de ellas $\Pi$ los valores de $u^{*}(t)$, para una sucesión 
infinita de momentos de tiempo $\tau_{1}, \tau_{2}, \ldots, \tau_{s}, \ldots$, son puntos interiores de la faceta $\Pi$ o de sus aristas. Entonces, por el lema 4.3, para cada momento de tiempo de esta sucesión la función de Pontryagin, como función de $u$, es constante en toda la faceta $\Pi$ o en alguna de sus aristas. Por lo tanto, existen dos puntos $u^{\prime}$ y $u^{\prime \prime}$, pertenecientes a una arista del poliedro $U$, en los cuales los valores de la función de Pontryagin coinciden para todos los momentos de tiempo de la sucesión $\tau_{1}, \tau_{2}, \ldots, \tau_{s}, \ldots$, es decir,

$$
\forall s, s=1,2, \ldots, \quad\left\langle\psi\left(\tau_{s}\right), B u^{\prime}\right\rangle=\left\langle\psi\left(\tau_{s}\right), B u^{\prime \prime}\right\rangle \quad \text { ó } \quad\left\langle\psi\left(\tau_{s}\right), B\left(u^{\prime}-u^{\prime \prime}\right)\right\rangle=0 .
$$

Denotando $B\left(u^{\prime}-u^{\prime \prime}\right)=a=\left(a_{1}, \ldots, a_{n}\right)^{T}$ obtenemos

$$
\forall s, \quad s=1,2, \ldots, \quad \sum_{k=1}^{n} a_{k} \psi_{k}\left(\tau_{s}\right)=0 .
$$

Puesto que $\psi(t)$ es solución del sistema lineal $\frac{d \psi}{d t}=-A^{T} \psi$ con coeficientes constantes, entonces cada componente $\psi_{k}(t)$ es una función de la forma $e^{\alpha_{k} t}\left[P_{k}(t) \cos \beta_{k} t+Q_{k}(t) \operatorname{sen} \beta_{k} t\right]$, donde $P_{k}(t)$ y $Q_{k}(t)$ son polinomios, y por eso la función $\varphi(t)=\sum_{k=1}^{n} a_{k} \psi_{k}(t)$ es analítica en $[0, T]$.

De la teoría de funciones de variable compleja se sabe que una función analítica que se anula en una sucesión de puntos convergentes a un punto interior del dominio, es identica a cero en su dominio de analiticidad. Por lo tanto, $\varphi(t) \equiv 0$, es decir, $\left\langle\psi(t), B\left(u^{\prime}-u^{\prime \prime}\right)\right\rangle \equiv 0$ en $[0, T]$. Pero entonces, por el lema 4.2, $B\left(u^{\prime}-u^{\prime \prime}\right)$ pertenece a un subespacio propio de $\mathbb{R}^{n}$ invariante respecto del operador $A$, lo que contradice la condición de posición general del poliedro $U$. De este modo se ha demostrado que sólo para un número finito de momentos de tiempo $t_{0}, t_{1}, \ldots, t_{r}$ los valores $u^{*}(t)$ se encuentran fuera de los vértices de $U$. de $U$

Ahora mostremos que $\forall i, i=1, \ldots, r, u^{*}(t)$ es constante en $\left(t_{i-1}, t_{i}\right)$ y toma su valor en algún vértice

En efecto:

Por hipotesis $u^{1}, \ldots, u^{N}$ son los vértices de $U$. Sea $M_{j}(j=1, \ldots, N)$ el conjunto de puntos $t \in\left(t_{i-1}, t_{i}\right)$, para los cuales $u^{j}$ maximiza la función de Pontryagin, es decir:

$$
F\left(u^{j}\right)=\operatorname{máx}_{u \in U} F(u), \quad \forall t \in M_{j} .
$$

De este modo $M_{1}, \ldots, M_{N}$ son conjuntos disjuntos (algunos de ellos pueden ser vacios) cuya unión es $\left(t_{i-1}, t_{i}\right)$.

Ahora mostremos que cada $M_{j}$ es abierto en $\left(t_{i-1}, t_{i}\right)$. Sea $t^{\prime} \in\left(t_{i-1}, t_{i}\right)$ un punto arbitrario de $M_{j}$. Entonces:

$$
\begin{aligned}
& \left\langle\psi\left(t^{\prime}\right), B u^{j}\right\rangle=\operatorname{máx}_{u \in U}\left\langle\psi\left(t^{\prime}\right), B u\right\rangle \\
& \left\langle\psi\left(t^{\prime}\right), B u^{k}\right\rangle<\left\langle\psi\left(t^{\prime}\right), B u^{j}\right\rangle, \quad \forall k \neq j, k=1, \ldots, N,
\end{aligned}
$$

pues:

$$
\begin{aligned}
\left\langle\psi\left(t^{\prime}\right), B u^{j}\right\rangle & =\operatorname{máx}_{u \in U}\left\langle\psi\left(t^{\prime}\right), B u\right\rangle \\
& >\left\langle\psi\left(t^{\prime}\right), B u^{k}\right\rangle, \quad \forall k \neq j, k=1, \ldots, N .
\end{aligned}
$$

Ya que cada una de las funciones $\left\langle\psi(t), B u^{j}\right\rangle, j=1, \ldots, N$, es continua sobre $\left(t_{i-1}, t_{i}\right)$, entonces existe un $\delta>0$ tal que:

$$
\left\langle\psi(t), B u^{j}\right\rangle>\left\langle\psi(t), B u^{k}\right\rangle, \forall t \in\left(t^{\prime}-\delta, t^{\prime}+\delta\right), \forall k \neq j, k=1, \ldots, N,
$$

es decir:

$$
\left\langle\psi(t), B u^{j}\right\rangle=\operatorname{máx}_{u \in U}\langle\psi(t), B u\rangle, \forall t \in\left(t^{\prime}-\delta, t^{\prime}+\delta\right),
$$

ya que, por lo probado anteriormente, los valores de $u^{*}(t)$ para cada momento de tiempo $t$ se encuentran en los vértices de $U$ excepto un número finito de ellos. Además, estamos trabajando en $\left(t_{i-1}, t_{i}\right)$ en el cual los valores de $u^{*}(t)$ están en los vértices de $U$.

Como $M_{j}$ es el conjunto de puntos $t \in\left(t_{i-1}, t_{i}\right)$ para los cuales $u^{j}$ maximiza la función de Pontryagin, entonces $\left(t^{\prime}-\delta, t^{\prime}+\delta\right) \subset M_{j}$. De este modo, para $t^{\prime} \in\left(t_{i-1}, t_{i}\right)$, se ha encontrado un radio $\delta>0$ tal que:

$$
\left(t^{\prime}-\delta, t^{\prime}+\delta\right) \subset M_{j}
$$


Por lo tanto, $M_{j}$ es abierto en $\left(t_{i-1}, t_{i}\right)$. Ya que $\left(t_{i-1}, t_{i}\right)=M_{1} \cup M_{2} \cup \cdots \cup M_{N}, M_{k} \cap M_{l}=\emptyset$ para $k \neq l$, y $\left(t_{i-1}, t_{i}\right)$ es conexo, entonces $M_{l} \neq \emptyset$ para algún $l$ y $M_{j}=\emptyset, \forall j \neq l, j=1, \ldots, N$. Así se tiene:

$$
\left(t_{i-1}, t_{i}\right)=M_{l}, \text { para algún } l \in\{1,2, \ldots, N\} .
$$

De esta manera, para todo $i, i=1, \ldots, r, u^{*}(t)$ es constante en $\left(t_{i-1}, t_{i}\right)$ y toma su valor en algún vértice de $U$.

5. Aplicaciones en mecánica. A continuación utilizamos los principios del máximo de Pontryagin indicados anterior para dar solución a un problema de control óptimo de origen mecánico.

Hay que conducir un vehiculo desde una posición de reposo inicial a otra posición de reposo final recorriendo una distancia $a$ en linea recta. Los controles disponibles para el conductor son el acelerador y el freno. La ecuación de movimiento del vehículo es:

$$
\frac{d^{2} x}{d t^{2}}=u
$$

de donde $u=u(t)$ representa la aceleración o desaceleración (frenado), y $x=x(t)$ la distancia recorrida en el instante $t$. El control $u$ puede tomar cualquier valor comprendido entre $-\alpha$ (frenado máximo) y $\beta$ (aceleración máxima), siendo $\alpha, \beta>0$. Se trata de trasladar el vehículo en el menor tiempo posible, es decir, se debe determinar el control óptimo y el tiempo minimo de transición del proceso.

\section{Solución :}

Transformemos la ecuación de movimiento en un sistema de ecuaciones diferenciales de primer orden. Se definen las variables:

$$
x_{1}=x, x_{2}=\frac{d x}{d t} .
$$

El problema a resolver es:

$$
\begin{array}{cl}
\min _{u} t_{f}(u) & \quad \text { [minimizar el tiempo utilizado] } \\
\text { sujeto a: }\left\{\begin{aligned}
\frac{d x_{1}}{d t}=x_{2} \text { [relación entre } x_{1} \mathrm{y} x_{2} \text { ] } \\
\frac{d x_{2}}{d t}=u \text { [ecuación de movimiento del vehículo] }
\end{aligned}\right. \\
x_{1}(0)=0 & \text { [la distancia recorrida al principio es cero] }, \\
x_{2}(0)=0 & \text { [la velocidad inicial es cero] }, \\
x_{1}\left(t_{f}\right)=a & \text { [la distancia total recorrida debe ser } a], \\
x_{2}\left(t_{f}\right)=0 & \text { [en el instante final la velocidad es cero] }, \\
-\alpha \leq u(t) \leq \beta & \text { [valores que puede tomar el control] }
\end{array}
$$

La función $H$ en este caso tiene la forma:

$$
H=\psi_{1} x_{2}+\psi_{2} u .
$$

Además, obtenemos el sistema de ecuaciones (ver ecuaciones (2.7) y (2.8)):

$$
\left\{\begin{array}{l}
\frac{d \psi_{1}}{d t}=0, \\
\frac{d \psi_{2}}{d t}=-\psi_{1},
\end{array}\right.
$$

para las variables auxiliares $\psi_{1}$ y $\psi_{2}$. Por lo tanto, $\psi_{1}(t)=A$ y $\psi_{2}(t)=B-A t$ ( $A$ y $B$ son constantes). Aplicando el Principio del Máximo de Pontryagin (teorema 4.2) [tomando en cuenta la condición $-\alpha \leq$ $u \leq \beta]$ :

$$
u^{*}(t)=\left\{\begin{array}{l}
-\alpha, \text { si } \psi_{2}<0, \\
\quad \text { cualquier valor entre }[-\alpha, \beta], \text { si } \psi_{2}=0, \\
\beta, \text { si } \psi_{2}>0 .
\end{array}\right.
$$


1. Si $u^{*}(t)=\beta$, las ecuaciones de estado son:

$$
\left\{\begin{array}{l}
\frac{d x_{1}}{d t}=x_{2} \\
\frac{d x_{2}}{d t}=\beta
\end{array}\right.
$$

La solución de dicho sistema es:

$$
\begin{aligned}
& x_{2}(t)=\beta t+C \\
& x_{1}(t)=\frac{\beta}{2} t^{2}+C t+D,
\end{aligned}
$$

la cual se puede expresar como:

$$
x_{1}(t)=\frac{1}{2 \beta}\left[x_{2}(t)\right]^{2}+\left(D-\frac{C^{2}}{2 \beta}\right),
$$

es decir:

$$
x_{1}=\frac{1}{2 \beta} x_{2}^{2}+K_{1}
$$

siendo $K_{1}$ una constante que depende de las condiciones iniciales. Representando, en este caso, las trayectorias de $x_{1}$ y $x_{2}$ en el plano de fase en la figura 5.1.

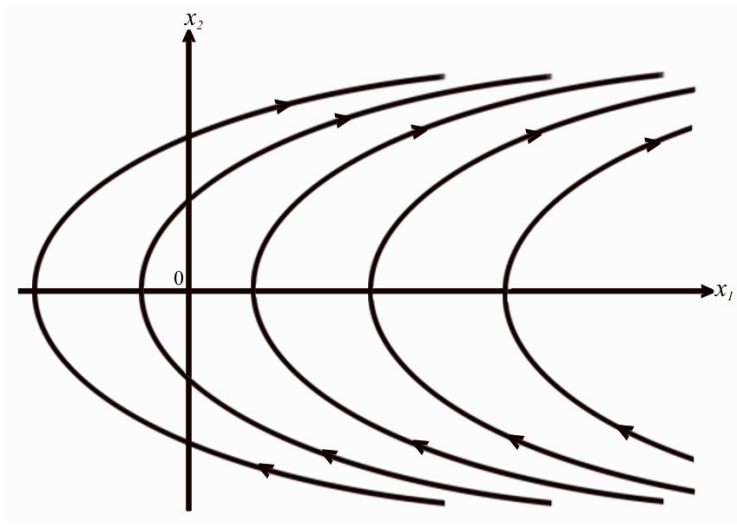

Figura 5.1: Trayectorias de $x_{1}$ y $x_{2}$ si $u^{*}(t)=\beta$.

Cada parábola corresponde a un valor de $K_{1}$. Las flechas indican el sentido de la evolución a través del tiempo que se obtiene teniendo encuenta lo siguiente:

(a) Como $\frac{d x_{2}}{d t}=\beta>0, x_{2}$ es creciente en el tiempo.

(b) Como $\frac{d x_{1}}{d t}=x_{2},\left\{\begin{array}{l}\text { será } \frac{d x_{1}}{d t}>0, \text { y por tanto } x_{1} \text { creciente en el tiempo si } x_{2}>0 \\ \text { será } \frac{d x_{1}}{d t}<0, \text { y por tanto } x_{1} \text { decreciente en el tiempo si } x_{2}<0 .\end{array}\right.$

2. Si $u^{*}(t)=-\alpha$, las ecuaciones de estado son:

$$
\left\{\begin{array}{l}
\frac{d x_{1}}{d t}=x_{2} \\
\frac{d x_{2}}{d t}=-\alpha
\end{array}\right.
$$

La solución del sistema es:

$$
\begin{aligned}
& x_{2}(t)=-\alpha t+E, \\
& x_{1}(t)=-\frac{\alpha}{2} t^{2}+E t+F,
\end{aligned}
$$


la cual se puede expresar como:

$$
x_{1}(t)=-\frac{1}{2 \alpha}\left[x_{2}(t)\right]^{2}+\left(F+\frac{E^{2}}{2 \alpha}\right)
$$

es decir:

$$
x_{1}=-\frac{1}{2 \alpha} x_{2}^{2}+K_{2}
$$

siendo $K_{2}$ una constante que depende de las condiciones iniciales. Representando, en este caso, las trayectorias de $x_{1}$ y $x_{2}$ en el plano de fase en la figura 5.2.

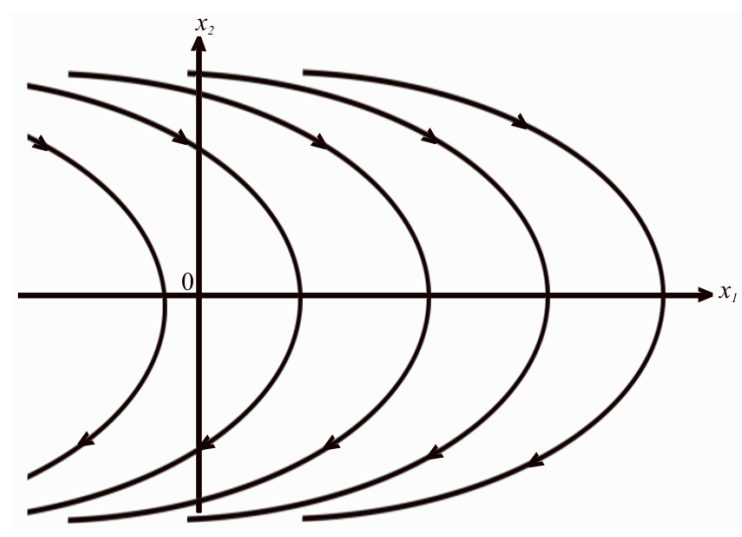

Figura 5.2: Trayectorias de $x_{1}$ y $x_{2}$ si $u^{*}(t)=-\alpha$.

Cada parábola corresponde a un valor de $K_{2}$. Las flechas indican el sentido de la evolución a través del tiempo que se obtiene tiniendo encuenta lo siguiente:

(a) Como $\frac{d x_{2}}{d t}=-\alpha<0, x_{2}$ es decreciente en el tiempo.

(b) Como $\frac{d x_{1}}{d t}=x_{2},\left\{\begin{array}{l}\text { será } \frac{d x_{1}}{d t}>0, \text { y por tanto } x_{1} \text { creciente en el tiempo si } x_{2}>0 \\ \text { será } \frac{d x_{1}}{d t}<0, \text { y por tanto } x_{1} \text { decreciente en el tiempo si } x_{2}<0 .\end{array}\right.$

Aunque hasta ahora hemos analizado por separado dos posibles valores para el control, puede ocurrir que dicho control tome un valor para ciertos valores de $t \mathrm{y}$ el otro valor para los restantes valores de $t$, por lo que ahora procedemos a considerar simultáneamente los dos tipos de trayectorias, es decir, procedemos a considerar las dos gráficas anteriores para definir un único gráfico, que aparece en la figura 5.3.

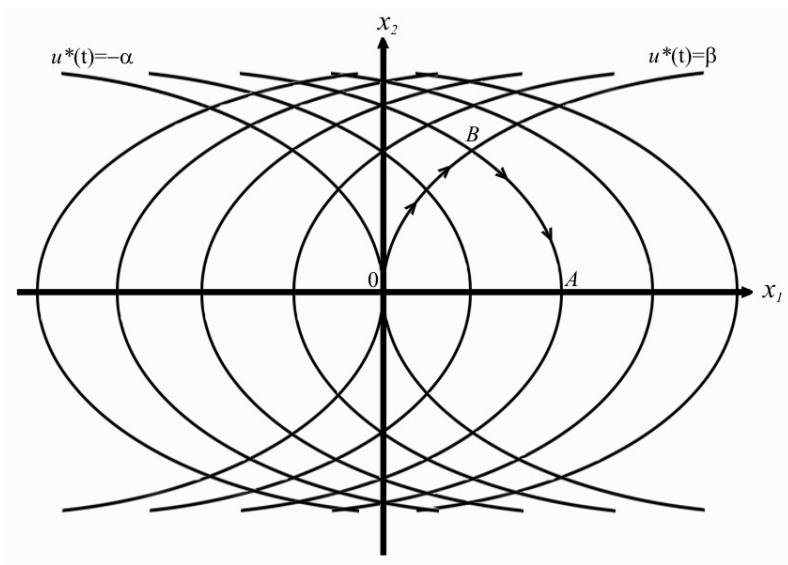

Figura 5.3: Solución óptima en el plano de fase.

Teniendo en cuenta las condiciones iniciales y finales del problema la trayectoria óptima será $O B A$, por lo que habrá que aplicar el control $u^{*}(t)=\beta$ (máxima aceleración) al principio, hasta cierto instante $t_{c}$ en el que se cambiará a $u^{*}(t)=-\alpha$ (máximo frenado), que ya se mantiene hasta el instante final. 
A continuación se calcula analíticamente el instante $t_{c}$ en el que cambia el control y el instante final $t_{f}\left(u^{*}\right)$

La ecuación de la parábola que contiene el tramo $O B$ se calcula teniendo en cuenta que corresponde al control $u^{*}(t)=\beta$, y que pasa por el punto $(0,0)$, por lo que se verifica:

$$
0=\frac{1}{2 \beta} 0^{2}+K_{1} \Rightarrow K_{1}=0 .
$$

Por tanto, su ecuación es:

$$
x_{1}=\frac{1}{2 \beta} x_{2}^{2} .
$$

La ecuación de la parábola que contiene al tramo $B A$ se calcula teniendo en cuenta que pasa por el punto $(a, 0)$, y que corresponde al control $u^{*}(t)=-\alpha$. De esto se obtiene:

$$
x_{1}=-\frac{1}{2 \alpha} x_{2}^{2}+a .
$$

Las coordenadas del punto $B$ se obtienen resolviendo el sistema

$$
\left\{\begin{array}{l}
x_{1}=\frac{1}{2 \beta} x_{2}^{2} \\
x_{1}=-\frac{1}{2 \alpha} x_{2}^{2}+a,
\end{array}\right.
$$

de donde:

$$
x_{2}=\sqrt{\frac{2 a \alpha \beta}{\alpha+\beta}}, x_{1}=\frac{\alpha a}{\alpha+\beta} .
$$

Como para $u^{*}(t)=\beta$ se había obtenido:

$$
x_{2}(t)=\beta t+C,
$$

y $x_{2}(0)=0$, resulta que:

$$
x_{2}^{*}=\beta t, \text { para } 0 \leq t \leq t_{c} .
$$

Particularizando en $t_{c}$ :

$$
x_{2}^{*}\left(t_{c}\right)=\beta t_{c}=\sqrt{\frac{2 a \alpha \beta}{\alpha+\beta}},
$$

de esto se tiene:

$$
t_{c}=\frac{1}{\beta} \sqrt{\frac{2 a \alpha \beta}{\alpha+\beta}}=\sqrt{\frac{2 a \alpha}{\beta(\alpha+\beta)}} .
$$

Calculemos ahora el instante final, es decir, el instante $t_{f}\left(u^{*}\right)$ en el que se alcanza el punto $A=(a, 0)$. En el tramo final se aplica el control $u^{*}(t)=-\alpha$. Ya se conoce que en tal caso es:

$$
x_{2}(t)=-\alpha t+E .
$$

Como $x_{2}^{*}\left(t_{f}\right)=0$, será $-\alpha t_{f}\left(u^{*}\right)+E=0$, por lo que $E=\alpha t_{f}\left(u^{*}\right), \mathrm{y}$

$$
x_{2}^{*}(t)=\alpha\left(t_{f}\left(u^{*}\right)-t\right), \text { para } t_{c} \leq t \leq t_{f}\left(u^{*}\right) .
$$

Ya que $x_{2}^{*}$ tiene que ser una función continua, será:

$$
x_{2}^{*}\left(t_{c}\right)=\alpha\left(t_{f}\left(u^{*}\right)-t_{c}\right)=\beta t_{c},
$$

por lo que el tiempo mínimo de transición del proceso es: 


$$
t_{f}\left(u^{*}\right)=\frac{\alpha+\beta}{\alpha} t_{c}=\frac{\alpha+\beta}{\alpha} \sqrt{\frac{2 a \alpha}{\beta(\alpha+\beta)}}=\sqrt{\frac{2 a(\alpha+\beta)}{\alpha \beta}}
$$

Por tanto, el control óptimo del proceso es:

$$
u^{*}(t)=\left\{\begin{array}{l}
\beta, \quad \text { si } 0 \leq t \leq \sqrt{\frac{2 a \alpha}{\beta(\alpha+\beta)}} \\
-\alpha, \quad \text { si } \sqrt{\frac{2 a \alpha}{\beta(\alpha+\beta)}}<t \leq \sqrt{\frac{2 a(\alpha+\beta)}{\alpha \beta}},
\end{array}\right.
$$

con la trayectoría óptima:

$$
x^{*}(t)=x_{1}^{*}(t)= \begin{cases}\frac{\beta}{2} t^{2}, & \text { si } 0 \leq t \leq \sqrt{\frac{2 a \alpha}{\beta(\alpha+\beta)}} \\ -\frac{\alpha}{2} t^{2}+\alpha \sqrt{\frac{2 a(\alpha+\beta)}{\alpha \beta}} t-a \frac{\alpha+\beta}{\beta}, & \text { si } \sqrt{\frac{2 a \alpha}{\beta(\alpha+\beta)}}<t \leq \sqrt{\frac{2 a(\alpha+\beta)}{\alpha \beta}},\end{cases}
$$

$\mathrm{y}$

$$
\frac{d x(t)}{d t}=x_{2}^{*}(t)= \begin{cases}\beta t, & \text { si } 0 \leq t \leq \sqrt{\frac{2 a \alpha}{\beta(\alpha+\beta)}} \\ -\alpha t+\alpha \sqrt{\frac{2 a(\alpha+\beta)}{\alpha \beta}}, & \text { si } \sqrt{\frac{2 a \alpha}{\beta(\alpha+\beta)}}<t \leq \sqrt{\frac{2 a(\alpha+\beta)}{\alpha \beta}}\end{cases}
$$

De esta manera se obtiene el proceso óptimo $\left(u^{*}(t), x^{*}(t)\right)$ del problema de tiempo mínimo expuesto, siendo el tiempo mínimo $T=t_{f}\left(u^{*}\right)$ el determinado lineas anteriores.

6. Conclusiones. Las conclusiones llegadas al realizar el presente trabajo son:

1. Se establecieron condiciones necesarias de optimalidad, llamado principio del máximo de Pontryagin como condición necesaria, para el problema de tiempo mínimo (3.3).

2. Se establecieron condiciones suficientes de optimalidad, llamado principio del máximo de Pontryagin como condición suficiente, para el problema de tiempo mínimo (3.3).

3. El principio del máximo de Pontryagin establece un método en la búsqueda de la solución del problema de tiempo mínimo (3.3).

4. Se utilizó el principio del máximo de Pontryagin en la búsqueda de la solución de un problema de control óptimo de origen mecánico, siendo el control óptimo una función por partes, cuyos valores son los extremos del intervalo dado.

ORCID and License

José Luis Ponte Bejarano https: / / orcid.org/0000-0002-4997-7950

Juan Carlos Ponte Bejarano https://orcid.org/0000-0002-8682-9682

Alexis Rodríguez Carranza https: / / orcid.org/0000-0002-0290-165X

Nelson Omar Aragones Salazar https://orcid.org/0000-0003-3031-8397

This work is licensed under the Creative Commons - Attribution 4.0 International (CC BY 4.0)

\section{Referencias}

[1] Intriligator DM. Mathematical optimization and economic theory. University of California. Prentice Hall. 2002. Pages 398-430.

[2] Kirk ED. Optimal control theory: An introduction. New York, Mineola. Prentice Hall. 2004. Pages 240-308.

[3] Hull GD. Optimal control theory for applications. New York. Springer Velag. 2003. Pages 89-91.

[4] Pontryagin LS, Boltyanskii VG, Gamkrelidze RV, Mischenko EF. The mathematical theory of optimal processes . Rusia. Interscience Publishers. 1962. Pages 115-188.

[5] Pontryagin LS. Optimal Control Processes. Selected Research Papers. 1959. Vol 1, pp 511-532.

[6] Cerdá TE. Optimización dinámica. España, Madrid. Prentice Hall. 2001. Pages 100-153. 